\title{
Corela
}

Cognition, représentation, langage

HS-19 | 2016

Le point de vue pris au mot

\section{Disqualifications et marqueurs de discours. Le cas de ma qué et otra que dans l'espagnol rioplatense}

\section{María Marta García Negroni}

\section{OpenEdition}

Journals

Édition électronique

URL : http://journals.openedition.org/corela/4395

DOI : $10.4000 /$ corela.4395

ISSN : $1638-573 \mathrm{X}$

Éditeur

Cercle linguistique du Centre et de l'Ouest - CerLICO

Référence électronique

María Marta García Negroni, «Disqualifications et marqueurs de discours. Le cas de ma qué et otra que dans l'espagnol rioplatense », Corela [En ligne], HS-19 | 2016, mis en ligne le 08 juin 2016, consulté le 20 avril 2019. URL : http://journals.openedition.org/corela/4395 ; DOI : 10.4000/corela.4395

Ce document a été généré automatiquement le 20 avril 2019

\section{(c) (i) (2)(2)}

Corela - cognition, représentation, langage est mis à disposition selon les termes de la licence Creative Commons Attribution - Pas d'Utilisation Commerciale - Partage dans les Mêmes Conditions 4.0 International. 


\title{
Disqualifications et marqueurs de discours. Le cas de ma qué et otra que dans l'espagnol rioplatense
}

\author{
María Marta García Negroni
}

\section{Introduction}

1 Dans le cadre des études sur la négation métalinguistique, il existe un terrain largement inexploré, à savoir celui des opérations de disqualification introduites par des marqueurs de discours. Dans cet article, nous nous proposons donc d'analyser les instructions argumentatives et polyphoniques (Ducrot 1984, 2004; Raccah 2005) inscrites dans la signification de ma qué et otra que, deux marqueurs spécialisés dans l'introduction de séquences ou de répliques disqualifiant un discours préalable. Notre approche relève d'un des courants de la linguistique française, celui des sémantiques instructionnelles dont la caractéristique est

qu'elles conçoivent la valeur sémantique des unités de langue comme des instructions pour construire, en situation, le sens des énoncés qui les utilisent. Elles se distinguent en cela d'une grande partie des autres approches en ce qu'elles différencient très nettement, dans leurs natures, la valeur sémantique des énoncés et celle des unités de langue. (Raccah et Várkonyi 2012:7)

Force est de constater que ces marqueurs n'ont fait l'objet d'aucune analyse dans les nombreux travaux qui, dans ces dernières années, se sont occupés des connecteurs, marqueurs ou particules de l'espagnol. Le seul antécédent pour notre étude repose sur l'entrée de dictionnaire qué va / van / vas / voy...iba / iban / ibas / iba a + Infinitif proposée par Santos Río (2003). Selon l'auteur, il s'agit d'un «schéma réactif permettant un refus catégorique de l'information positive apportée par l'interlocuteur » (2003: 545). Comme nous le verrons plus loin, cette structure possède en effet certains points en commun avec les énoncés dans lesquels figure ma qué. Spécifiques à l'espagnol rioplatense et à l'usage assez fréquent aussi bien dans l'interaction orale «colloquiale» que dans la 
langue écrite la reproduisant ou l'imitant, les marqueurs ma qué et otra que nous renvoient à l'italien, langue en contact avec l'espagnol parlé en Argentine sous la grande vague d'immigration de la fin du XIX et au début du XX $\mathrm{XX}^{\mathrm{e}}$ siècle ${ }^{1}$. Ainsi, dans l'expression ma qué - tout comme dans une autre formule typiquement argentine, jma si!, permettant d'exprimer l'acceptation par résignation ou par fatigue -, on reconnaît clairement la présence de la conjonction adversative italienne $m a$ (pero en espagnol ; mais en français) et derrière otra que se cache certainement l'expression emphatique positive de l'italien altroché, de laquelle le marqueur argentin est un calque. Dans (1) et (2), respectivement tirés d'un article d'opinion au fort registre familier et d'un message publié sur un site web, l'utilisation de l'un ou l'autre de ces deux marqueurs est bien illustrée :

(1) Estamos atareados en este egocentrismo argentino. En este egotismo de cada uno actuando su propio default económico, su propio padecimiento. Somos una conjunción de egoísmos. Ya nadie habla del diálogo auspiciado por la Iglesia. Ma qué diálogo : monólogo, soliloquio, monodia, monomanía.

http://www.lanacion.com.ar/210269-autobiografia-de-un-pais-egocentrico

[Cet égocentrisme argentin nous bouffe la vie. Chacun, dans son narcissisme individuel, relate sa propre crise économique, sa propre souffrance. Nous sommes un tas d'égoïstes réunis, car plus personne ne dialogue ou ne parle d'un même son de cloche, celui instauré par l'Église. Dialogue, tu parles! Monologue, monisme, unicité, monomanie.]

(2) Dieta. Otra que Reduce Fat Fast. Taringa! (después de este título, aparece una dieta)

www.taringa.net/posts/.../Dieta---Otra-que-Reduce-Fat-Fast-_.html

[Régime. Bien mieux que Reduce Fat Fast. Taringa! (Un régime apparaissant sous ce titre)]

Dans ce qui suit et à travers l'analyse de données provenant de divers sites Internet et d'échantillons de discours réels argentins, nous essayerons de mettre en évidence l'étroite relation qu'entretiennent ces deux marqueurs avec les mécanismes de disqualification et de rectification dans le discours. En premier lieu, nous rappellerons les propriétés sémantiques de la négation métalinguistique dans son emploi méta-discursif (section 2.). Ensuite, nous nous arrêterons sur les disqualifications introduites par ma qué et nous distinguerons celles dans lesquelles le segment dans la portée du marqueur est un mot ou un syntagme du discours d'autrui de celles dans lesquelles ce segment reprend la totalité de l'énoncé de l'interlocuteur (section 3.). Cette distinction nous permettra d'introduire quelques réflexions à propos de la valeur évidentielle du futur périphrastique, forme verbale avec laquelle, dans les répliques avec ma qué, on reprend généralement, pour le disqualifier, le discours de l'autre. Finalement, nous étudierons les structures introduites par otra que (section 4.). Notre objectif est de montrer que, bien que les deux marqueurs introduisent un point de vue présenté comme provenant d'un autre discours et qu'ils autorisent, tout comme la négation méta-discursive, la disqualification de ce point de vue, la configuration polyphonique et argumentative de l'énonciation mise en place dans chaque cas diffère selon que la disqualification est introduite par l'un ou l'autre des deux marqueurs, ce qui détermine des lectures clairement différenciées. Enfin, dans la dernière partie (section 5.), nous reprenons en guise de conclusion les résultats de l'analyse.

\section{Négation méta-discursive, disqualification et répliques introduites et par ma qué et otra que}

3 Comme on le sait, dans le cadre de sa conception polyphonique du sens - cadre avec lequel la sémantique des points de vue élaborée par Pierre-Yves Raccah $(2005,2012)$ est 
intimement liée -, Ducrot distingue trois types de négation : la polémique, la descriptive et la métalinguistique. Si la négation descriptive est analysée comme un dérivé délocutif de la négation polémique et permet de «représenter un état de choses sans que son auteur présente sa parole comme s'opposant à un discours adverse » (1984 : 216-217), la polémique suppose toujours la présence de deux points de vue opposés et internes au propre discours. Ces points de vue sont attribués à des êtres discursifs différents, les énonciateurs $E_{1}$ et $E_{2}$ : le premier est le responsable du point de vue positif sous-jacent, le deuxième constitue le rejet du premier et constitue la perspective que le locuteur adopte. En ce qui concerne la négation métalinguistique, Ducrot la caractérise comme étant une négation contredisant les termes mêmes d'une parole effective qu'elle prétend réfuter et, dans ce sens, elle n'oppose pas deux énonciateurs, mais deux locuteurs différents. Pour le linguiste, c'est dans le cadre de cette réfutation d'un locuteur adverse que la négation métalinguistique présente certaines caractéristiques particulières, à savoir :

- elle est capable de refuser les présupposés d'un énoncé antérieur ;

- elle peut avoir, à la place de l'effet habituellement descendant ou abaissant, une valeur ascendante. Selon Ducrot, il est possible de dire 'Pierre n'est pas intelligent, il est génial', mais uniquement en réponse à un locuteur qui a effectivement qualifié Pierre d'intelligent.

Selon l'auteur, ce sont précisément ces deux propriétés qui la distinguent clairement de la négation polémique, dont l'effet est toujours abaissant (i.e., lecture «moins que ») et qui conserve toujours les présupposés de l'énoncé positif sous-jacent.

Or, tout comme nous avons tenté de le démontrer dans un travail antérieur (García Negroni 2009), la négation métalinguistique ne contredit pas toujours les termes mêmes d'une parole effective préalable et elle n'a jamais l'effet abaissant ou descendant de la négation polémique. Ce qui caractérise cette négation c'est qu'elle disqualifie l'espace ou cadre de discours ${ }^{2}$ imposé par une parole antérieure (explicite ou évoquée dans le discours la disqualifiant) et qu'elle déclare donc se situer dans un cadre différent de celui qui est rejeté ou disqualifié. Et cela qu'il s'agisse de son emploi métalinguistique proprement dit (c'est-à-dire, des cas dans lesquels la négation met en question l'emploi d'un terme ou d'une séquence de mots de l'interlocuteur en vertu d'une règle syntaxique, morphologique, sociale, etc. mise en évidence par l'énoncé postérieur qui introduit la rectification), ou qu'il s'agisse d'un emploi méta-discursif, dans lequel la négation disqualifie une énonciation préalable ou évoquée comme telle dans l'énonciation même. C'est précisément avec cet emploi méta-discursif qu'un rapport peut être établi dans le cas des répliques introduites par ma qué et otra que, objet de ce travail.

Définie comme une négation qui disqualifie le cadre d'un discours préalable, explicite ou évoqué dans l'énonciation qui la contient, la négation méta-discursive présente les propriétés suivantes:

- Dans la mesure où elle s'oppose à un dire préalable (ou présenté comme tel) qu'elle prétend réfuter, son énonciation montre deux locuteurs différents (3) ou un même locuteur à des moments différents, i.e., en tant que locuteur et ex-locuteur (4).

(3) A : Juan vino ayer.

$B$ : No, no vino.

[A : Jean est venu hier.

B : Non, il n'est pas venu.]

(4) Creo que había dicho que João era brasileño. Bueno, no es brasileño, sino portugués.

[Je crois que je t'avais dit que João était Brésilien. Bon, il n'est pas Brésilien, mais 
Portugais.]

- Dans la mesure où elle disqualifie un cadre de discours préalable, la négation méta-discursive peut nier aussi bien le point de vue posé (5) que le point de vue présupposé, cadre de discours à la lumière duquel s'interprètent les contenus posés dans l'énoncé positif sous-jacent (6) :

(5) No, Pedro no dejó de fumar. Acabo de verlo prender un cigarrillo.

[Non, Pierre n'a pas arrêté de fumer. Je viens de le voir allumer une cigarette.]

(6) No, Pedro no dejó de fumar. De hecho, nunca fumó en su vida.

[Non, Pierre n'a pas arrêté de fumer. En fait, il n'a jamais fumé de sa vie.]

- En ce qui concerne les effets déclenchés par l'énonciation de la négation métadiscursive, et dans la mesure où, à la différence de la négation polémique, elle ne dit pas refuser de s'inscrire dans l'espace qu'elle met en scène (Anscombre 1990), mais disqualifie le cadre de discours imposé par une parole antérieure, cette négation n'a jamais l'effet abaissant ou réducteur, caractéristique de la négation polémique : son effet est ou bien contrastif (lecture « contraire à ", (7)), ou bien majorant (lecture " plus que », (8)), ou bien différenciateur (lecture « différent de », (9)) :

(7) A : - Juan es inteligente.

B : - No, no es inteligente. Es un tarado.

[A : - Jean est intelligent.

B : - Non, il n'est pas intelligent. C'est un imbécile.]

(8) A : - Juan es inteligente.

B : - No, no es inteligente. ¡Es súper inteligente !

[A : - Jean est intelligent.

$\mathrm{B}:-$ Non, il n'est pas intelligent. Il est très intelligent !]

(9) A : - Juan es inteligente.

B : - No, no es inteligente. (A lo sumo / en todo caso) es astuto.

[A : - Jean est intelligent.

B : - Non, il n'est pas intelligent. (Au mieux) il est malin.]

- La négation méta-discursive apparaît normalement suivie d'un énoncé correctif qui introduit la rectification assumée par le locuteur. Destiné à remplacer le segment dans la portée de la négation, cet énoncé de correction ou de rectification ne s'avère pas obligatoire lorsque l'effet est contrastif ( $\left.7^{\prime}\right)$, ou lorsque la négation ne prétend pas annuler un contenu présupposé, mais seulement celui qui est exposé (5'). Par contre, il est obligatoire dans les autres cas (6), (8), et (9) : la présence de l'énoncé correctif y est nécessaire pour indiquer quelle est l'interprétation désirée ${ }^{3}$.

(7') A : - Juan es inteligente.

B : - No, no es inteligente. $<<$ Es un tarado.>>

[A : - Jean est intelligent.

B : - Non, il n'est pas intelligent. $<<$ C'est un imbécile >>]

5'. No, Pedro no dejó de fumar. $<<$ Acabo de verlo prender un cigarrillo. $>>$

[Non, Pierre n'a pas arrêté de fumer. $\ll<$ Je viens de le voir allumer une cigarette >>]

- La négation méta-discursive se caractérise par un contour intonatif spécifique d'emphase (Horn 1985, 1989 ; Forest 1992 ; Fillmore 1985 ; Nølke 1992, 1994), lequel, bien que non obligatoire, favorise souvent l'interprétation, surtout dans les cas où l'énoncé négatif n'est pas suivi de la rectification ${ }^{4}$.

Maintenant, si dans sa forme typique (Girotti 2000), la négation méta-discursive peut assumer, selon le cas, n'importe laquelle des structures suivantes :

- Non + énoncé négatif + énoncé correctif

- Non + énoncé négatif

- Non + énoncé correctif

- Non

7 il est intéressant de constater que, dans certains contextes, d'autres constructions peuvent apparaître à la place de ces structures. Tel est le cas des répliques introduites en espagnol par (Pero) si, dans lesquelles la protase en si fonctionne comme un indice de la disqualification ou de la mise en question du cadre de discours antérieur, et cela dans la 
mesure où cette protase constitue une justification de la mise en question qui reste élidée (García Negroni 2002) et des séquences introduites par ma qué et otra que, dont nous traiterons dans ce qui suit.

\section{Ma qué, rejet absolu du cadre de discours préalable et les effets 'contraire à' et 'différent de'}

Dans l'ensemble, on peut dire que par ma qué $P$ le locuteur disqualifie et rejette un segment de discours antérieur $(P)$, qu'il réitère, si $P$ a été énoncé précédemment par l'interlocuteur, ou qu'il évoque simplement dans sa propre énonciation en le reprenant d'un autre discours préalable. Ainsi, dans la mesure où l'énonciation de ma qué $P$ montre que $P$ a été dit précédemment, on peut affirmer que le marqueur ma qué constitue, au même titre, par exemple, que la conjonction espagnole à valeur évidentielle que (Rodriguez Ramalle 2008, 2014), l'une des manifestations de l'expression de l'évidentialité de l'espagnol's.

Par ailleurs, et tout comme la négation méta-discursive, ma qué $P$ est normale bien que pas nécessairement suivie (ou précédée, dans certains cas) d'un énoncé correctif $Q$, avec lequel le locuteur s'identifie. Cet énoncé explicite le nouveau cadre de discours qui, selon le locuteur, correspond à la meilleure représentation discursive de la situation dont il s'agit, ou qui, à défaut, présente un argument justifiant le rejet de $P$ et orientant ainsi vers le nouveau cadre de discours qui sera adopté par la suite. À l'instar de la négation métadiscursive, ce nouveau cadre peut être soit le contraire du précédent (effet contrastif), soit, tout simplement, un autre différent (effet différenciateur). En outre, et tout comme dans le cas de la négation méta-discursive, la présence de l'énoncé correctif ou rectificatif $Q$ n'est obligatoire que dans le deuxième cas (c'est-à-dire, lorsque le nouveau cadre constitue un espace différent de celui qui est disqualifié) et cela pour faire expliciter la lecture adéquate selon le locuteur.

\subsection{Ma qué $\mathrm{P}$ : quand le segment $\mathrm{P}$ est un mot ou un syntagme du discours d'autrui}

Prenons pour commencer l'exemple suivant, où $m a$ qué $P$ apparaît en début de réplique, $P$ reprenant les termes mêmes d'une parole effective antérieure ${ }^{6}$ :

(10) A :- Pero usted daba el OK.

B : - (Tono agudo) ¿Ma' que OK ? Yo no podía decir nada, no podía decir ni que sí ni que no.

Corpus oral personnel, 5

[A : - Mais vous avez donné votre accord.

B : - (aigu) Quel accord ? Je n'ai même pas pu dire ni oui ni non ${ }^{7}$.]

Comme on peut le constater en (10), le locuteur B rejette énergiquement l'accusation de A en mettant en question au moyen de Ma qué $O K$, l'application de (dar el) $O K$ (« donner son accord ») à la situation dont ils parlent. Ainsi, et malgré le fait que ma qué porte sur un seul mot, le rejet n'est pas ici métalinguistique mais clairement méta-discursif, puisqu'il disqualifie l'énonciation de l'interlocuteur dans sa totalité. En effet, afin de renforcer sa position discursive, le locuteur introduit l'énoncé correctif $Q$ (Je n'ai même pas pu dire ni oui ni non), avec lequel il s'identifie et qui est proposé comme le seul cadre valide pour la suite de l'échange verbal. Autrement dit, et tel que l'indique la sémantique des points de vue, les instructions fournies par les unités de langue (dans le cas de (10), « donner l'accord »; « dire ni 
oui ni non ») constituent « des contraintes sur la manière de voir la situation dont parlent les énoncés utilisant ces unités de langue » (Raccah et Várkonyi 2012 : 7).

11 Mais, ainsi que nous l'avons annoncé, de manière analogue à la négation méta-discursive, il n'est pas obligatoire que le segment $P$ dans la portée de ma qué ait été énoncé immédiatement auparavant par l'interlocuteur dans le dialogue. Ce segment peut être repris (sous la forme de discours rapporté direct ou indirect) dans le discours où il se trouve disqualifié, comme en (11) et (12), ou encore évoqué de sorte que c'est l'énonciation de ma qué $P$ même qui oblige à le récupérer dans l'interdiscours (c'est le cas de (13), (14) et (15)).

(11) Todas las mujeres tienen algo. Yo estoy enamorado de una que me trastornó la vida. Yo tengo 32 y ella 20. ¡Me volvió loco ! Mi vieja, no sabés las cosas que me está diciendo: "Es una nena, es una nena”. “Ma qué nena! Pesa más de 30 kilos”, le digo. Una uva es. Una uvita. Una torrontés. Es increíble. Somos unos nenes con las mujeres.

http://www.lanacion.com.ar/211182-entrevista-al-hombre-comun

[Toutes les femmes ont un petit quelque chose. Je suis amoureux d'une fille qui a chamboulé ma vie. J'ai 32 ans et elle en a 20. Elle m'a rendu fou! Tu ne sais pas tout ce que me dit ma mère : "C'est une gamine, c'est une môme! ». "Tu parles d'une gamine! Elle pèse plus de 30 kilos », je lui dis. C'est un grain de raisin. Un nectar. Un Torrontés. C'est incroyable. Nous sommes vraiment des mômes avec les femmes.]

Dans (11) et par l'énonciation de ¡Ma qué nena! («tu parles d'une gamine!»), le locuteur montre son désaccord profond avec la qualification nena proposée par sa mère (qualification reprise ici sous la forme du discours direct rapporté : « C'est une gamine, c'est une môme!») pour caractériser la personne dont le locuteur est amoureux. Celui-ci disqualifie donc $P$ et, dans l'énoncé rectificatif suivant $Q$, il introduit la justification de son refus de $P$ (Pesa más de 30 kilos, « elle pèse plus de 30 kilos») amenant ainsi son discours vers la conclusion contraire, i.e. «c'est une femme».

Dans (12), en revanche, l'énonciation de ¡Ma qué relax! ("Quoi? Me détendre?») disqualifie le discours précédemment mentionné sous forme de discours rapporté au style indirect (le sugerimos que se tome su tiempo para relajarse, "nous lui avons suggéré de prendre son temps pour se détendre »):

(12) Cuando llega -retrasado por el tráfico impiadoso de Buenos Aires-, y le sugerimos que se tome su tiempo para relajarse, Donato nos contesta con un : “ $\mathrm{Ma}$ qué relax !", en el mismo tono entusiasta que se impondrá a lo largo del encuentro. http://www.espacioliving.com/1263623-cocina-a-la-italiana-el-paraiso-de-donatode-santis

[Quand il est arrivé, retardé par la circulation de Buenos Aires, et nous lui avons suggéré de prendre son temps pour se détendre, Donato nous a répondu par un « Quoi ? Me détendre?!» avec le même ton enthousiaste qu'il conservera durant tout l'entretien.]

13 Enfin, comme l'illustrent (13) et (14), c'est le discours ma qué $P$ même qui oblige à récupérer dans l'interdiscours ce qui est disqualifié. Ainsi, (13), qui constitue le titre d'un article de journal signé par le journaliste Pepe Eliaschev,

(13) ¿Ma qué "inseguridad” ? La opinión de Pepe Eliaschev blogs.perfil.com/eliaschev/2010/08/16/¿ma-que-inseguridad, 16/8/10

[Comment ça « insécurité » ? L'avis de Pepe Eliaschev] son désaccord. En effet, et comme le signale le journaliste plus loin dans son article, sous 
l'étiquette " insécurité », c'est la violence criminelle en Argentine qui est cachée dans ces discours :

(13a) Uno de los eufemismos clásicos de la Argentina es hablar de falta de seguridad.

[C'est vraiment un euphémisme en Argentine de dire que nous manquons de sécurité.]

Fortement disqualifié par ¿Ma qué "inseguridad" ? (Comment ça "insécurité »?), ce cadre de discours est remplacé, dans l'article, par un autre, pleinement assumé cette fois-ci par le locuteur, car plus approprié pour représenter l'état actuel des choses : il ne s'agit pas d'insécurité ou de manque de sécurité, mais de criminalité et de manque de responsabilité de la part du gouvernement :

(13b) En todo caso, no hay falta de seguridad, hay exceso de criminalidad.

[Dans tous les cas, ce n'est pas un manque de sécurité qu'il y a, mais trop de crimes.]

De même, dans le fragment (14) ci-dessous, le locuteur disqualifie, au moyen de ma qué, le segment $P$ (gripe ni gripe) bien que celui-ci ne soit pas énoncé immédiatement auparavant. Le locuteur reprend ici une information journalistique diffusée au moment de l'envoi de ce twitt selon laquelle la Présidente argentine (nommée ici CFK) était grippée. Le locuteur démontre son refus de croire à cette information (ma qué grippe ni grippe, la grippe, mon œil !) et la retouche à sa manière en attribuant la cause momentanée de l'alitement de CFK (quedó de cama, elle en est restée malade) aux fortes critiques du journaliste Jorge Lanata.

(14) \#cfk vio el programa del genio de Lanata el domingo y quedó de cama ! ma que gripe ni gripe jajajajajajajajajajaj

twitter, @ Luismomberg,22/5/12

[\#CFK a regardé l'émission de Lanata et en est restée malade! Elle a la grippe, la grippe, mon œil ! (rires).]

Notons que, dans (14), l'énoncé de rectification $Q$ avec son effet différenciateur (c'est-àdire la véritable explication de l'absence de CFK, selon le locuteur) précède la mise en question et la disqualification de $P$, ce qui fait une autre similitude avec la négation métadiscursive. Effectivement, même si l'ordre n'est pas des plus communs, la structure "rectification + disqualification " s'avère également possible dans les énoncés avec négation méta-discursive, comme, par exemple, dans (15):

(15) Somos kirchneristas, no alperovichistas

http://cafeprensa.blogspot.com.ar/2011/11/somos-kirchneristas-no-

alperovichistas.html

[Nous sommes kirchneristes, pas alperovichistas.]

Enfin, dans l'exemple (16), c'est également l'énonciation de ma qué $P$ même qui évoque le segment $P$ de la doxa (El tango es patrimonio de la humanidad, Le tango appartient au patrimoine mondial). Celui-ci est rejeté et remplacé par $Q$ (Nuestro, nuestro y nada más que nuestro, Il est à nous, à nous et rien qu'à nous), cadre de discours représentant l'opposé de P.

(16) TANGO : ¿iMá qué patrimonio de la humanidad ?! Nuestro, nuestro y nada más que nuestro

El escritor Ariel Corbat dejándose llevar por su corazón rebelde se resiste, algo en serio y algo en broma, a que el Tango sea considerado patrimonio de la humanidad. http://www.igooh.com/notas/tango-ma-que-patrimonio-de-la-humanidadnuestro-nuestro-y-nada-mas-que-nuestro/, 4/3/09

[TANGO : Patrimoine mondial! N'importe quoi ! Il est à nous, à nous et rien qu'à nous. 
L'écrivain Ariel Corbat emporté par son tempérament rebelle résiste à imaginer, sur le ton de la blague et de la gravité, que le Tango soit considéré comme patrimoine mondial.] pas rare de les retrouver combinées avec la particule négative ni, qui reprend le segment $P$ (comme, par exemple, dans (14)) ou qui introduit un autre segment $P^{\prime}$, également rejeté. Dans de tels contextes, les constructions du type qué $P$ ni $P$ ' sont également possibles, car, comme le précise Santos Rio (2003: 470), elles expriment un sentiment général de « rejet teinté de surprise et de reproche ». Selon l'auteur, $P$ ' peut être égal à $P$ (jqué camiseta ni qué camiseta!), un morphème nominal de genre contraire à $P$ (;Qué bocadillo ni qué bocadilla!) ou encore un syntagme, ici niño muerto (¿Qué piano ni qué niño muerto!). Ainsi, et de manière semblable à ce que l'on peut constater dans les répliques introduites par (Pero) si (Montolio 1999a, 1999b; Garcia Negroni 2002), la présence de ma (pero, en espagnol) précédant qué $P$ ni qué $P$ renforce la valeur d'opposition et de refus déjà existante par rapport au point de vue adverse évoqué dans le discours.

Ainsi, dans (17), et compte tenu de la disqualification introduite par ma qué, ce ne sont ni Marx ( $P$ ) ni Nietzsche ( $\left.P^{\prime}\right)$ qui seront au centre de ce premier post, bien que ces deux philosophes puissent, dans le cadre des sciences sociales et humaines, être considérés comme des référents appropriés pour un blog appelé «Bon Sens ». Comme l'évoquent l'énoncé rectificatif $Q$ et le premier paragraphe du post, tous deux permettant au locuteur d'introduire un nouveau cadre discursif pour la suite de son discours, c'est du groupe de rock heavy metal La Renga qu'il sera question.

(17) Ma' que Marx ni que Nietzsche. Acá, La Renga.

Damas y caballeros, dando comienzo al estreno formal de este pequeño rinconcito que hemos dado en llamar "Sentido Apropiado" -y mostrando un primer ejemplo de los "asuntos" que podrán ser objeto de este blog, así como del general modus operandi del autor del mismo- hoy tenemos preparado un humilde, pequeño, limitado y muy personal análisis de un tema que ya todos han de conocer...

Damas y caballeros, hoy tenemos el orgullo de presentarle a Uds. a... ¡La Renga !

http://sentidoapropiado.blogspot.com.ar/2009/02/ma-que-marx-ni-que-nietszcheaca-la.html, 2/2/09

[Il n'y a pas de Marx ni de Nietzsche qui soit. Ici, c'est La Renga.

Mesdames et Messieurs, en guise de première publication officielle sur ce blogounet que nous appellerons «Bon Sens » et afin de vous donner un premier aperçu des «problèmes » qui seront traités dans ce blog, ainsi que le mode opératoire de son auteur, nous avons concocté une modeste, simple, limitée mais néanmoins personnelle analyse d'un sujet déjà connu de tous...

Mesdames et Messieurs, aujourd'hui nous sommes fiers de vous présenter ... La Renga!]

Pour la même raison, on peut également trouver ma qué $P$ suivi de la locution à polarité négative de haut degré ou surréalisante ni (qué) ocho cuartos (Garcia Negroni 1997), qui souligne le profond désaccord du locuteur avec le cadre de discours $P$ dans la portée de ma qué. C'est le cas dans ce fragment (18), titre d'un blog dans lequel on évoque, ironiquement, le probable discours d'un réalisateur de cinéma ne souhaitant plus travailler avec des acteurs réels.

(18) Ma que memoria emotiva, ni que ocho cuartos

Una vez haciendo unas pruebas para un corto experimental (yo actor) dirigido por un amigo y su socio, el segundo en un momento dijo (mitad en joda, mitad en serio) : "no veo la hora de que se perfeccionen las animaciones para no tener que trabajar más con actores". 
http://paraisosartificiales.blogspot.com.ar/2007/02/ma-que-memoria-emotiva-yocho-cuartos.html

[De la mémoire émotive, mon œil !

Après avoir fait un jour (en tant qu'acteur) des essais pour un court métrage indépendant géré par un ami et son associé, ce dernier me dit à un moment (en ne plaisantant qu'à moitié) : «Vivement qu'on fasse de superbes animations virtuelles pour qu'on arrête de travailler avec les acteurs ».]

En définitive, soit que le segment $P$ (ou l'enchaînement $P$ ni $P^{\prime}$ ) disqualifié est effectivement prononcé par l'interlocuteur, soit qu'il est évoqué dans l'énonciation même, ma qué constitue une marque de rejet énergique du locuteur face à ce discours d'autrui. Ce rejet est habituellement suivi par un énoncé de rectification, souvent postposé, qui présente le point de vue (contraire à celui qui est refusé ou différent de lui) avec lequel le locuteur s'identifie et qui, par conséquent, est proposé comme unique appréciation valide pour la suite du discours ou de l'échange. Toutefois, si cet énoncé correctif n'est pas toujours nécessaire (rappelons qu'il l'est uniquement lorsqu'il indique une lecture "différent de» pour expliquer quelle est la lecture adéquate selon le locuteur), l'énonciation peut parfois renvoyer à la réinterprétation du segment précédent disqualifié. À titre d'exemple, nous pouvons examiner le fragment (19), dans lequel le locuteur B (un homme politique argentin) se défend d'une attaque de A (un journaliste) concernant les critiques unilatérales de son parti sans jamais proposer de solutions concrètes :

(19) A : - Pero al final ustedes critican mucho al Gobierno, pero no proponen nada.

No dicen nada de cómo se soluciona esta situación.

B : - Pero yo lo he dicho. Lo he dicho en ese discurso que usted tanto me ha censurado.

A : - ¿Ma qué "censurado" ? Lo he mostrado.

Corpus oral personnel, 4.

[A : - Mais en fin de compte, vous critiquez beaucoup le gouvernement, mais vous ne proposez rien. Vous ne préconisez rien pour résoudre cette situation.

$B$ : - Mais je l'ai déjà fait. Je l'ai dit dans ce discours que vous m'avez tant censuré.

A : - «Censuré » ? Ça ne va pas, non ! Je l'ai montré.]

Comme on peut le constater, dans sa réponse à $\mathrm{A}$, le locuteur $\mathrm{B}$ affirme avoir proposé des solutions « dans ce discours que vous m'avez tant censuré ». En effet, dans l'émission de télévision d'où ce dialogue est extrait, le journaliste avait critiqué à plusieurs reprises la façon dont B avait traité, quelques jours plus tôt, ses adversaires. Or, dans sa réplique, A reprend le mot «censuré» prononcé antérieurement par B et au moyen de son énonciation introduite par ma qué invalide l'application de ce terme à la situation dont il s'agit. Mais dans les faits, A laisse sous-entendre bien autre chose. En introduisant, dans la rectification $Q$, le segment lo he mostrado (je l'ai montré) comme cadre de discours contraire à he cesurado su discurso ("j'ai censuré votre discours»), il force à la réinterprétation du segment $P$ (García Negroni 2000). En d'autres termes, l'énonciation de $Q$ exige une autre lecture du terme censuré, différente du sens qu'il présentait dans le discours de B. En effet, si dans l'intervention de B, censuré avait le sens de 'critiqué', dans celle de A (et, par relecture, dans celle de B aussi), le sens sélectionné est 'coupé' ou encore 'mutilé. Ainsi, l'énonciation de la correction $Q$ permet d'introduire une double disqualification du discours de B. En effet, le fait de remettre en question la pertinence d'un terme, présenté comme erroné au moment de caractériser la situation, permet d'ajouter un second discrédit aux dires de $B$ : en vertu de la rectification $Q$, il passe de surcroît pour un menteur. 


\subsection{Ma qué $P$ : quand le segment $P$ est un énoncé complet de l'autre}

d'un mot (un nom : gamine, détente, insécurité, grippe, ou bien par un participe : censuré) ou un syntagme nominal (patrimoine mondial, la mémoire émotionnelle), mais $P$ peut aussi reprendre un énoncé complet, comme dans (20) et (21) :

(20) A : - tan raro es que te ofrezcan algo gratis?

B : - nooo... ma que va a ser raro!

http://www.purogeek.com/foro/threads/servidor-smtp-\%C2\%BFpropio-o-

externo.10614/

[A : - C'est si bizarre que ça qu'on t'offre quelque chose gratuitement?

B : - Bizarre ! nooon ... pas du tout !]

(21) Ma qué va a estar viendo jugadores! Está calculando los billetes que les va a chorear a los turcos estos. (Comentario de Pepe a la nota periodística "Veo, veo, ¿qué ves?”, en cuya bajada se afirma : "Maradona estuvo en la cancha viendo a los jugadores que va a dirigir").

http://www.ole.com.ar/futbol-internacional/Veo-veo-ves_0_493750817.html?

commentsPage $=9$

[Il vient regarder jouer les footballeurs! Tu parles! Il est plutôt en train de calculer la tune qu'il va leur piquer qu'autre chose. (Commentaire de Pepe à l'article de la chronique "Veo, veo, ¿qué ves?", où figure en pied: "Sur le terrain, Maradona est venu regarder jouer les footballeurs qu'il entraînera »)]

Notons, toutefois, que, comme dans les structures réactives qué va / van / vas / voy... iba / iban / ibas / iba a + Inf (Santos Río 2003), P subit une modification : le verbe prend ici la forme du futur périphrastique, manquant évidemment à sa valeur prospective habituelle ${ }^{8}$ . De ce fait, comment doit-on interpréter la présence de cette périphrase dans les énoncés avec ma qué?

Dans un travail récent sur la relation entre le futur et l'évidentialité, Escandell Vidal (2010) émet l'hypothèse que le futur simple ne codifie pas la localisation temporelle des événements, mais une signification évidentielle: ce temps aurait ainsi comme signification de base l'indication que la source de ce qui est communiqué est interne au sujet parlant: "le seul moyen permettant au sujet parlant d'accéder au contenu propositionnel communiqué est fourni par un processus interne d'inférence» (2010:26). En ce sens, le futur, ainsi que le conditionnel, serait un moyen de mettre en place « un microsystème de distinctions évidentielles ": là où le futur ne transmettrait que des informations obtenues par inférence du locuteur, le conditionnel indiquerait que la source est une rumeur. Selon les termes de l'auteur, «dans les deux cas, l'évidence dont dispose le sujet parlant est indirecte : inférentielle dans le premier cas, citative dans le second» $(2010: 22)$. En ce qui concerne la forme du futur périphrastique ir $+a+$ infinitif, succinctement traitée, l'auteur déclare que, sur le même modèle que le futur, elle n'aurait pas «la localisation temporelle des événements à venir comme faisant partie de sa signification de base» $(2010: 29)$ : elle acquerrait ces valeurs prospectives seulement de manière inférentielle.

L'hypothèse selon laquelle le contenu sémantique de type évidentiel constitue la signification de base du futur semble séduisante pour expliquer de façon unifiée les emplois prospectifs (usages prescriptifs, directifs et de compromissifs) et conjoncturels du futur. Cependant, elle n'explique pas du tout les emplois concessifs du type será muy inteligente, pero no lo parece (peut-être qu'il est très intelligent, mais il ne le montre pas) en 
réponse à l'affirmation Es un chico muy inteligente (C'est un garçon très intelligent), car l'évidence tient ici de l'ordre de la citation plutôt que de l'inférence9.

D'un point de vue polyphonique, la présence du futur dans les énoncés concessifs peut toutefois facilement être expliquée. Son énonciation indique que le point de vue mis en scène est un point de vue d'autrui (soit de l'interlocuteur, comme dans l'exemple cidessus, soit d'un collectif, comme dans le titre de la pièce de théâtre No seré feliz, pero tengo marido (Peut-être que je ne suis pas heureuse, mais j'ai un mari), que le locuteur accepte de reprendre l'espace d'un instant, afin d'introduire un autre point de vue, qui sera effectivement adopté plus tard dans son discours. Et tout mène à penser que c'est par cette configuration polyphonique que se dessine la différence avec l'utilisation du futur périphrastique dans les réfutations ou disqualifications comme (20) et (21).

En effet, alors que l'occurrence du futur simple signale que le point de vue mis en scène est pris en considération par l'interlocuteur - pour le valider totalement (emploi prospectif du futur, du style No matarás, Tu ne tueras point), ou bien pour le modaliser (emploi conjoncturel du style Serán las 11, Il est peut-être 11 heures), ou encore simplement pour le concéder (emploi concessif, du style Será muy inteligente, pero no lo parece, Peut-être qu'il est très intelligent, mais il ne le montre pas) -, l'énonciation du futur périphrastique dans son emploi non prospectif doit, en revanche, être mise en rapport avec le fait que le point de vue évoqué est toujours un point de vue d'autrui que le locuteur rejette catégoriquement et dont il se distancie dès le début : non seulement il ne l'assume pas, mais il ne l'envisage même pas pour l'accepter ou le concéder dans son discours. Et cette lecture évidentielle (de citation rejetée) n'est pas seulement déclenchée dans de tels cas, où le futur périphrastique apparaît introduit par le marqueur évidentiel ma qué. Sans sa valeur prospective habituelle, la périphrase de futur s'emploie également dans des contextes où l'on souhaite montrer le rejet d'un point de vue d'autrui. À cet égard, nous pouvons comparer (22) et (23), d'une part, avec (24) et (25), d'autre part.

(22) ¿Vos le creés ? Mirá si va a trabajar de sol a sol, como dice.

[Tu le crois? Tu parles! qu'il travaille du matin au soir, comme il dit.]

(23) ¡¿Qué va a ser complicada ? ¡Si es divina María!

Comment ça, elle est pénible ?! C'est un ange Marie !]

(24) Mirá si será inútil este tipo. ¡Ni un huevo pasado por agua sabe hacer!

[Ce type, vraiment un bon à rien. Il ne sait même pas faire cuire un œuf à la coque !]

(25) ¡Ay, esta María! ¡Si será complicada!

[Pour être compliquée, elle est compliquée Marie !]

Comme nous pouvons le constater, (24) et (25) affichent un verbe au futur. L'énonciation est prise en charge par un locuteur qui assume comme propre le point de vue selon lequel este tipo (ce type) est qualifié de inútil (un bon à rien) ou Maria de complicada (compliquée), et ce à partir de certains éléments de la situation de discours qui argumentent en faveur de ces points de vue (Escandell Vidal dirait que la source de ce qui est communiqué par le sujet parlant serait son processus interne d'inférence). Dans (22) et (23), qui contiennent des formes au futur périphrastique, le locuteur affiche, à l'inverse, son profond désaccord avec ce qui a été affirmé auparavant par quelqu'un d'autre (cf. l'incise como dice (comme il dit) dans (22)) ou avec le point de vue assumé antérieurement par l'interlocuteur ((23) pourrait en effet être la réplique du locuteur à son interlocuteur ayant affirmé que María es complicada, Marie est pénible).

31 De cette manière, les configurations polyphoniques mises en place, respectivement, par l'utilisation du futur ou de la périphrase de futur expliquent clairement la présence de cette dernière dans les énoncés avec ma qué. Étant spécialisé dans la disqualification d'un 
discours d'autrui (i.e. provenant d'une source citée ou évoquée dans l'énoncé qui le met en question), ma qué signale toujours un fort rejet de la part du locuteur du point de vue introduit. C'est ce que nous pouvons constater dans des cas comme (20), où B s'oppose avec énergie au point de vue présupposé par l'énonciation interrogative de A (es raro que te ofrezcan algo gratis, c'est bizarre qu'on t'offre quelque chose gratuitement), ou comme (21), dans lequel le commentaire conteste vigoureusement la caractérisation de la situation proposée par le journaliste (Maradona estuvo en la cancha viendo a los jugadores que va a dirigir, Sur le terrain, Maradona est venu regarder jouer les footballeurs qu'il entraînera).

\section{Otra que, disqualification d'un point de vue antérieur et effet ascendant}

Que se passe-t-il avec les disqualifications introduites par otra que? Il est vrai que dans cette structure, le locuteur remet également en question un discours antérieur, $P$, et s'identifie avec le point de vue présenté dans $Q$. Mais, contrairement à ce qui se passe avec les énoncés introduits par ma qué, la configuration polyphonique mise en place par otra que n'implique pas que le locuteur se situe dans un cadre de discours contraire ou différent de celui qui est rejeté. Avec otra que $P$, la disqualification concerne son propre discours antérieur - voire un point de vue d'autrui, mais accepté aussi par le locuteur luimême - et cela dans la mesure où il est insuffisant pour caractériser la situation dont il parle. De ce fait, nous pouvons affirmer que les énoncés avec otra que ne sont pas compatibles avec la présence de la périphrase de futur et que l'interprétation implique un changement de perspective énonciative du locuteur, s'auto-corrigeant lui-même pour aller au-delà ou avec plus de force argumentative dans le même sens que P. Ainsi, même s'il partage avec ma qué la possibilité d'introduire un point de vue présenté comme procédant d'une source citée, otra que peut aussi porter sur un point de vue qui se présente comme venant du propre discours antérieur du locuteur. Dans les deux cas, l'effet de son énonciation demeure toujours amplificateur ou ascendant (lecture «plus que »). Ainsi, en (2), repris ici, et (26) :

(2) Dieta. Otra que Reduce Fat Fast. Taringa !

[Régime. Bien mieux que Reduce Fat Fast. Taringa !]

(26) Otra que Larissa : la polaca que se saca todo. Así como la vedette paraguaya se hizo famosa en todo el mundo por sus fotos provocativas durante el Mundial de Sudáfrica, la modelo polaca fue la gran sensación de la Euro.

[Larissa, c'est rien à coté ! la Polonaise enlève tout. Tout comme la star Paraguayenne devenue mondialement connue grâce à ses photos provocantes durant la coupe du monde de football en Afrique du Sud, le mannequin Polonais a fait sensation durant la coupe de l'Euro.]

Nous remarquerons que, dans les deux cas, le locuteur se distancie du cadre de discours $P$, mais pas en raison de son total désaccord avec celui-ci ( $P$ aurait pu être un point de vue qu'il aurait lui-même précédemment accepté). Si $P$ est abandonné au profit du segment $Q$, qui suit et qui sera le point de vue adopté par le locuteur dans la suite du discours, c'est parce que $P$ est jugé trop faible maintenant du point de vue argumentatif. Ainsi, disqualifiés par otra que car insuffisants, les discours el suplemento dietario Reduce Fat Fast ayuda a adelgazar (le complément alimentaire Reduce Fat Fast vous aide à perdre du poids) dans (2), ou las fotos provocativas de la vedette paraguaya Larissa son las más osadas (les photos provocantes de la star paraguayenne Larissa sont les plus osées) dans (26) seront 
remplacés lors de la rectification $Q$ par des discours plus forts et allant au-delà dans le même sens que $P$. Dans le cas (2), c'est davantage la communauté virtuelle Taringa!, plus que le complément alimentaire Reduce Fat Fast, qui nous offre la recette miracle pour perdre du poids. Dans le cas (26), l'attention est davantage portée sur le mannequin polonais qui ose enlever tout, plus que sur Larissa.

En somme, l'énonciation de otra que $P$ n'implique pas, de la part du locuteur, le rejet absolu de $P$ pour lui substituer un cadre de discours contraire ou différent. Si $P$ est disqualifié avec otra que c'est uniquement parce qu'il est devenu insuffisant pour l'argumentation que le locuteur cherche à soutenir. Prenons par exemple, le cas de quelqu'un qui, faisant des éloges sur le dîner que j'ai préparé, énonce (27) :

(27) Otra que Mallmann...

[Mallmann c'est rien à côté...

Il ne serait pas convenable de comprendre ici que la personne en question estime que Francis Mallmann, un célèbre cuisinier argentin, cuisine mal. Avec (27), le locuteur ne rejette pas l'idée que Mallmann est un bon cuisinier; il insiste plutôt sur le fait qu'il apprécie ma cuisine. En ce sens, une paraphrase possible de (27) serait « affirmer que ta cuisine est aussi bonne que celle de Mallmann serait peu dire ». De même dans (28),

(28) Otra que Tevez

Así como Carlos Tevez se negó a jugar en Manchester City, Samuel Eto'o hizo lo mismo con la selección de Camerún. Con el agravante de convencer a sus compañeros para no presentarse a jugar un amistoso ante Argelia. El Comité disciplinario sancionó al ex Barcelona con 15 partidos de suspensión.

[Plus fort que Tevez

Tout comme Carlos Tevez, qui s'est refusé à jouer à Manchester, Samuel Eto'o en a fait de même avec l'équipe du Cameroun. À ces griefs s'ajoute le fait d'avoir tenté de convaincre ses compagnons de ne pas jouer un match amical contre l'Algérie. Le Comité de discipline a sanctionné l'ancien de Barcelone de 15 matchs de suspension.]

le segment $P$ (Tevez) n'est pas rejeté comme cadre de discours pour le remplacer par un autre différent ou par son contraire. Introduit par otra que, $P$ est montré comme insuffisant pour décrire la situation dont il est question (i.e. l'attitude du buteur africain Eto'o et la sanction infligée). À cet égard, les marqueurs comparatifs d'égalité (así como, tout comme; hizo lo mismo, en a fait de même) et de supériorité (con el agravante, à ces griefs) s'avèrent très parlants : Eto'o n'a pas uniquement fait quelque chose à la manière de Tevez,

(28a) Así como Carlos Tevez se negó a jugar en el Manchester, Samuel Eto’o hizo lo mismo con la selección de Camerún,

[Tout comme Carlos Tevez qui s'est refusé à jouer à Manchester, Samuel Eto'o en a fait de même avec l'équipe du Cameroun,]

mais est allé bien plus loin dans ses agissements :

(28b) Con el agravante de convencer a sus compañeros para no presentarse a jugar ante Argelia.

[À ces griefs s'ajoute le fait d'avoir tenté de convaincre ses compagnons de ne pas jouer un match amical contre l'Algérie.]

Clairement différentes des énonciations avec ma qué, les disqualifications faites par le biais de otra qué n'ont jamais la prétention de se placer dans un cadre de discours différent ou opposé à celui qui est rejeté. Si otra que rejette $P$, c'est parce que $P$, point de vue assumé ou accepté précédemment par le locuteur lui-même, ne suffit plus à l'argumentation. Par conséquent, le segment de rectification $Q$, qui suit, prend la forme d'une autocorrection à l'effet ascendant. Ainsi, et avec cette valeur affirmative et 
ascendante (lecture "plus que $P »)$, otra que se réconcilie avec la valeur d'origine de l'expression italienne altroché (cf. - C'era gente ? - Altroché!).

\section{En guise de conclusion}

Ce travail nous a permis d'étudier les configurations argumentatives et polyphoniques mises en place par deux marqueurs de l'espagnol rioplatense, ma qué et otra que, spécialisés dans les opérations de disqualification.

Tout d'abord, nous avons tenté de souligner les liens sémantiques étroits rencontrés entre les répliques de disqualification introduites par ces marqueurs et la négation métadiscursive, négation qui se caractérise par la disqualification d'un cadre de discours préalable.

Deuxièmement, nous avons cherché à expliquer les différentes lectures déclenchées par la présence de l'un ou l'autre de ces deux marqueurs et ce, malgré les similitudes qui les rapprochent. Rappelons qu'ils sont tous les deux fréquents dans l'interaction «colloquiale» orale de l'espagnol rioplatense, ainsi que dans la langue écrite qui la reproduit ou l'imite; qu'ils introduisent un segment $P$ qui reprend ou qui évoque un discours antérieur qu'ils disqualifient; et que l'énonciation dans laquelle ils apparaissent est très fréquemment suivie d'un énoncé de rectification $Q$ avec lequel le locuteur s'identifie.

Donc, si dans les deux cas, la fonction primordiale des répliques qu'ils introduisent consiste à instaurer un nouveau cadre de discours présenté par le locuteur comme étant le seul approprié pour la caractérisation argumentative de la situation dont on parle, l'énonciation de ma qué $P$ est toujours liée au refus absolu de $P$, ce qui autorise une lecture " contraire à » ou "différent de ", donnant lieu à un éloquent et manifeste conflit conversationnel. De son côté, l'énonciation de otra que $P$ présente simplement un changement de perspective énonciative du locuteur, s'auto-corrigeant afin de pousser encore plus loin (lecture "plus que ») sa logique de négation face à ce qui est mis en question: si $P$ est disqualifié par le locuteur, ce n'est pas parce qu'il s'agit d'un point de vue d'autrui uniquement évoqué pour être rejeté (en fait, comme nous l'avons vu, $P$ peut être un point de vue préalablement assumé ou accepté par le locuteur). S'il se trouve disqualifié, c'est uniquement parce qu'il est considéré comme insuffisant pour l'appréhension argumentative de la situation.

Enfin, l'analyse présentée a fourni des preuves concernant l'interprétation évidentielle citative de certains emplois du futur périphrastique en espagnol (i.e. dans les énonciations de disqualification avec ma qué et dans les autres structures de réfutation ici considérées). En effet, dans ces emplois, l'énonciation du futur périphrastique est nécessairement liée à la mise en scène d'un point de vue provenant toujours d'une source citée que le locuteur reprend uniquement pour le rejeter. 


\section{BIBLIOGRAPHIE}

Aikhenvald Alexandra, Evidentiality, Oxford, Oxford University Press, 2004.

Anscombre Jean-Claude, "Thèmes, espaces discursifs et représentation événementielle », in Anscombre Jean-Claude et Gino Secondo Zaccaria (comp.), Fonctionnalisme et pragmatique, Milan, Unicopli, 1990, pp. 43-150.

Bermúdez Fernando, "Los tiempos verbales como marcadores evidenciales. El caso del pretérito perfecto compuesto", Estudios Filológicos, n 40, 2005, pp. 165-188.

Ducrot Oswald, Le dire et le dit, Paris, Minuit, 1984.

Ducrot Oswald, “Sentido y argumentación”, in Elvira Narvaja de Arnoux et García Negroni María Marta (eds), Homenaje a Oswald Ducrot, Buenos Aires, Eudeba, 2004, pp. 359-370.

Escandell Vidal María Victoria, "Futuro y evidencialidad", Anuario de lingüística hispánica, $\mathrm{n}^{\circ} 26$, 2010, pp. 9-34.

Fillmore Charles, "Frames and the Semantics of Understanding", Quaderni di Semantica, vol. VI, $\mathrm{n}^{\circ}$ 2, 1985, pp. 222-251.

Fontanella de Weinberg María Beatriz (coord.), El español de la Argentina y sus variedades regionales, Buenos Aires, Hachette, 2000.

Fontanella de Weinberg María Beatriz, El español bonaerense. Cuatro siglos de evolución lingüística, Buenos Aires, Hachette, 1987.

Forest Robert, «L'interprétation des énoncés négatifs », Langue française, n 94, 1992, pp. 35-47.

García Negroni María Marta, «Les notions de Déréalisation / Réalisation / Surréalisation et la classification des Locutions à Polarité Negative », Revista de Filología Francesa, n 10, 1997, pp. 81-95.

García Negroni María Marta, “Acerca de los fenómenos de relectura y reinterpretación en el discurso", Discurso y Sociedad, vol. 2 n 4, 2000, pp. 89-108.

García Negroni María Marta, « Disqualification, confirmation et (représentation du) discours de l'autre. À propos des répliques introduites par ¡Pero si... ! ", Cahiers de linguistique française, $\mathrm{n}^{\circ} 24$, 2002, pp. 243-264.

García Negroni María Marta, “Negación y descalificación. A propósito de la negación metalingüística", Ciências e Letras, nº 45, 2009, pp. 61-82.

Girotti Elsa, Negación metalingüística y rectificación, Thèse de Master de l'Universidad Nacional de Cuyo, 2000.

Horn Lawrence, "Metalinguistic Negation and Pragmatic Ambiguity", Language, vol. LXI, $\mathrm{n}^{\circ} 1$, 1985, pp. 121-174.

Horn Lawrence, A Natural History of Negation, Chicago, University of Chicago Press, 1989.

Leonetti Manuel et Escandell Vidal María Victoria, “On the Quotative Readings of Spanish Imperfecto", Cuadernos de Lingüística, vol. X, 2003, pp. 135-154. 
Montolío Estrella, "Las construcciones condicionales", in Bosque Ignacio et Demonte Violeta (eds), Gramática descriptiva de la lengua española, Madrid, Espasa, 1999a., pp. 3643-3737.

Montolío Estrella, “ ¡Si nunca he dicho que estuviera enamorada de él! Sobre construcciones independientes introducidas por si con valor replicativo", Oralia, n² 2, 1999b, pp. 37-70.

Nølke Henning, «Ne...pas : négation descriptive ou polémique ? Contraintes formelles sur son interprétation », Langue Française, $n^{\circ}$ 94, 1992, pp. 48-67.

Nølke Henning, Le regard du locuteur, Paris, Kimé, 1994.

Raccah Pierre-Yves, «Une sémantique du point de vue : de l'intersubjectivité à l'adhésion », in «L'Énonciation identitaire : entre l'individuel et le collectif », dir. par Danielle Forget, Discours social, vol. XXI, 2005, pp. 205-242.

Raccah Pierre-Yves et Várkonyi Zsófia, « “Assez”, c'est presque "trop” », in Neveu Franck, Muni Toke Valelia, Blumenthal Peter, Klingler Thomas, Ligas Pierluigi, Prévost Sophie et TestonBonnard Sandra (eds), $3^{e}$ Congrès Mondial de Linguistique Française, Lyon, 4-7 juillet 2012, ILF / EDP Sciences, (Publié sur CD ROM), 2012.

Reyes Graciela, Los procedimientos de cita : citas encubiertas y ecos, Madrid, Arco Libros, 1994.

Rodríguez Ramalle Teresa, “Sobre marcadores y su relación con la modalidad evidencial”, in García Negroni María Marta (ed.) Marcadores del discurso : perspectivas y contrastes, Buenos Aires, Santiago Arcos, 2014, pp. 233-251.

Rodríguez Ramalle Teresa, "Estudio sintáctico y discursivo de algunas estructuras enunciativas y citativas del español”, Revista Española de Lingüística Aplicada (RESLA), n² 21, 2008, pp. 269-288.

Santos Río Luis, Diccionario de partículas, Salamanca, Luso-Española de Ediciones, 2003.

\section{NOTES}

1. L'espagnol du Río de la Plata montre de fortes empreintes de l'italien, surtout au niveau lexical et prosodique. Pour des études sur l'incidence de l'immigration italienne sur l'espagnol du Río de la Plata, voir par exemple les travaux de Fontanella de Weinberg $(1987,2000)$.

2. Nous utiliserons ce terme dans le sens d'Anscombre (1990).

3. L'énoncé correctif est aussi obligatoire dans le cas de la négation métalinguistique proprement dite (A : -La ropa me cabió bien en la maleta. B : -No, no te CABIÓ bien. Te CUPO bien./ A : - Les vêtements sont bien entrés dans la valise. B : - Non, ils ne sont pas bien ENTRÉs. (À la limite) Ils sont bien RENTRÉS). Il en va de même pour le morphème intonatif d'emphase sur le terme disqualifié par la négation et sur la rectification.

4. On remarquera, tout de même, que le morphème intonatif d'emphase peut ne pas être présent. C'est ce qui se passe, en particulier, dans le cas des énoncés de la forme 'négation méta-discursive + sino + rectification' (João no es brasileño sino portugués, así que no creo que pueda ayudarte mucho con tu tarea sobre la historia del Brasil. João n'est pas brésilien mais portugais : je ne crois pas qu'il pourra t'aider avec ton travail sur l'histoire du Brésil).

5. L'évidentialité est souvent définie comme la catégorie grammaticale qui exprime l'existence d'une source ou l'origine de l'information communiquée tout en précisant le type de source (directe ou indirecte) dont il s'agit (Aikhenvald 2004). Contrairement à d'autres langues dans lesquelles cette catégorie, en plus d'être grammaticalement codifiée, demeure obligatoire, l'espagnol est, quant à lui, souvent classé comme une langue n'ayant pas grammaticalisé ces signifiés car - affirme-t-on - il ne possède pas de morphèmes verbaux faisant référence à la source d'information. Cependant, comme cela a déjà été démontré (Reyes 1994 ; Bermúdez 2005 ; 
Leonetti et Escandell Vidal 2003 ; Escandell Vidal 2010, entre autres), au moins trois morphèmes verbaux existent en espagnol (le futur, le conditionnel et l'imparfait) qui, dans certains empois, affichent une interprétation évidentielle claire dans la mesure où leur énonciation indique que la source du point de vue introduit est indirecte (soit par inférence, soit par citation). À côté de ces formes verbales, auxquelles nous proposons d'ajouter le futur périphrastique (cf. ci-dessous section 3.2.), l'espagnol possède aussi des marqueurs de discours - parmi lesquels se trouve ma qué - qui peuvent être analysés comme des formes qui, de manière intrinsèque, codifient une signification évidentielle.

6. À la différence de la structure réactive qué va / van / vas / voy...iba / iban / ibas / iba a + Infinitif analysé par Santos Río (2003), le segment de discours introduit et disqualifié par ma qué peut être un seul et unique mot.

7. Etant donné qu'il s'agit exclusivement d'une particule de la langue orale, il y a des variations en ce qui concerne la manière dont elle est reproduite à l'écrit. Même si la forme la plus fréquente est ma qué, nous la trouverons également déclinée sous les formes má qué, ma' que, ma' qué, ma que.

8. Comme pour toutes les structures qué va / van / vas / voy...iba / iban / ibas / iba a + Infinitivo, le verbe ir du futur périphrastique dans ma qué $P$ peut se décliner soit au présent (voy / vas / van, etc.), soit à l'imparfait (iba / ibas / iban, etc.). Selon Santos Río (2003 : 545), les formes du présent seront davantage exploitées pour les situations présentes ou futures non conditionnées et pour certaines situations non conditionnées du passé (- Es que tiene frío. - Qué va a tener frío ; - Al parecer están enfermas. - Qué van a estar enfermas ; - Ese llegará lejos. - Qué va a llegar lejos; - Dicen que ya ha muerto. - Qué va a haber muerto). Les formes de l'imparfait, quant à elles, seront utilisées quand il y a des conditionnements (- Te hubiera vencido yo. - Qué me ibas a haber vencido, etc.).

9. Selon l'auteur, la valeur concessive du futur « naît d'une situation paradoxale : l'interlocuteur dit quelque chose et l'émetteur le répète au futur. Ainsi, le même contenu propositionnel est présenté à la fois comme contenu factuel (dont la vérification du locuteur est immédiate) mais aussi en tant que contenu non factuel (dont la vérification du locuteur n'est pas directement accessible). Ceci indique que le sujet parlant B n'accepte pas l'affirmation préalable de l'interlocuteur, et ne tient pas compte de l'information qui a été communiquée comme étant une information acceptable. En ayant recours au futur, B oblige à interpréter ce contenu comme étant une donnée non vérifiée, contredisant clairement et directement les propos tenus par l'interlocuteur. Ce décalage provoque une lecture concessive, dans laquelle est éliminée (ou au moins remise en question) la factualité de ce qui est communiqué et on lui retire sa force dans l'efficacité de la chaîne argumentative » (2010 : 27).

\section{RÉSUMÉS}

Dans cet article, nous nous proposons d'analyser les instructions argumentatives et polyphoniques inscrites dans la signification de ma qué et otra que, deux marqueurs spécialisés dans l'introduction de séquences ou de répliques disqualifiant un discours préalable. Nous signalons d'abord les liens qui peuvent être établis avec la négation méta-discursive; puis, nous proposons une description polyphonique et argumentative qui cherche à rendre compte des différences que ces marqueurs, propres de l'espagnol du Rio de la Plata, présentent par rapport aux discours disqualifiés. Nous montrons ainsi, d'une part, que l'énonciation de ma qué $P$ est toujours liée au refus absolu de $P$, donnant lieu à un éloquent et manifeste conflit 
conversationnel. De l'autre, que l'énonciation de otra que P disqualifie un point de vue préalable (du locuteur ou d'autrui), de telle sorte que la rectification qui suit peut être lue comme une autocorrection avec un effet toujours majorant.

In this paper, polyphonic-argumentative instructions conveyed by two markers specialized in disqualification operations, ma qué and otra que, are analyzed. While having their links with metadiscursive negation pointed, a polyphonic-argumentative description accounting for the differences these typically River Plate colloquial markers show as regards the discourse they disqualify and the rectification they enable is proposed. As a conclusion, it can be said that the evidential marker ma qué strongly rejects someone else's previous speech, thus highlighting a conversational conflict. Otra que, in contrast, disqualifies a speaker's or someone else's - accepted by the speaker - preliminary point of view, which can be read as a self-correcting discourse with an upward effect.

INDEX

Mots-clés : Marqueur de discours, disqualification, rectification, évidentialité, futur périphrastique

Keywords : Discourse marker, evidentiality, periphrastic future 